\title{
Intrathyroidal Thymus (Incidentaloma) Mimicking Thyroid Neoplasia in DICER1 Syndrome
}

\author{
Marek Niedziela ${ }^{a}$ Nelly Sabbaghian ${ }^{b}$ Leanne de Kock ${ }^{b}$ c William D. Foulkes ${ }^{\text {b, c }}$ \\ a Department of Pediatric Endocrinology and Rheumatology, Poznan University of Medical Sciences,

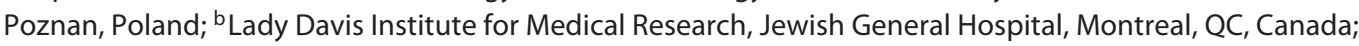 \\ 'Department of Human Genetics, McGill University, Montreal, QC, Canada
}

\section{Established Facts}

- Thyroid incidentalomas, i.e., "nonpalpable" thyroid nodules, in children and adolescents are common, and both benign and malignant lesions may be found [1].

- Thymic inclusions and ectopic intrathyroidal thymus with a similar ultrasonographic pattern to papillary thyroid carcinoma should be considered in the differential diagnosis of an apparent thyroid nodule $[1,2]$.

- Thyroid disease, particularly multinodular goiter (MNG), is the most frequent feature of DICER1 syndrome caused by loss-of-function germline DICER1 alterations on chromosome 14q32.13; MNG in DICER1 syndrome is usually represented by benign nodules, but thyroid cancer may also occur.

\section{Novel Insights}

- Intrathyroidal ectopic thymic tissue can be mistaken for a thyroid nodule and may lead to unnecessary surgery and/or invasive diagnostic procedures in children, especially if it coexists with another organ tumor as observed in DICER1 syndrome.

- Thyroid incidentalomas in DICER1 syndrome patients should be viewed with great suspicion, but not all thyroid disorders are DICER1-related in these patients, nor are they necessarily related to a malignant process.

\section{Keywords}

Thyroid incidentaloma · Intrathyroidal thymus · DICER1 syndrome

\section{Abstract \\ Introduction: With the use of ultrasonography for the evalu- ation of thyroid and nonthyroid neck diseases, the incidental discovery of previously unsuspected thyroid nodules/non-}

palpable lesions has increased. Intrathyroidal thymus arises due to aberrant thymic migration during embryogenesis. It is thought to be rare, fulfils the classic criteria for diagnosis of an incidentaloma and it can be mistaken for a thyroid nodule. Multinodular goiter is the main endocrine manifestation of DICER1 syndrome, a tumor predisposition syndrome. Careful thyroid examination including ultrasound is thus necessary for DICER1 syndrome patients. Case Report: A 4-year-old prepubertal boy was referred to the pediatric en- 
docrinology department following an earlier diagnosis of pleuropulmonary blastoma (PPB) type III, which is a hallmark component of DICER1 syndrome. The patient underwent surgery followed by chemotherapy. Genetic analysis identified a germline DICER1 pathogenic variant (c.2062C $>$ T, p.R688*) in the child and in 5 relatives. A second somatic DICER1 RNase IIIb hotspot mutation (c.5438A>G, p.E1813G) was identified in DNA extracted from the proband's PPB. He had no goiter on clinical examination, but a heterogenous, well-delineated, avascular, solid and hypoechogenic lesion with pseudomicrocalcifications was observed in the right lobe $(4.1 \times 3.1 \times 2.6 \mathrm{~mm})$, suggestive of thyroid neoplasia. Diagnostic work-up and a 3-year follow-up excluded thyroid neoplasia and confirmed the diagnosis of an intrathyroid benign thymic lesion. Discussion and Conclusion: This case exemplifies that thyroid incidentalomas in DICER1 syndrome patients should be viewed with great suspicion, but that not all thyroid disorders are indeed DICER1-related in these patients.

(C) 2020 European Thyroid Association Published by S. Karger AG, Basel

\section{Introduction}

With the use of ultrasonography for the evaluation of thyroid and nonthyroid neck diseases, the incidental discovery of previously unsuspected thyroid nodules/nonpalpable lesions has increased. An intrathyroidal thymus arises due to aberrant thymic migration during embryogenesis, is thought to be rare and it fulfils the classic incidentaloma criteria. The thymus has a unique appearance on ultrasonography, and ectopic thymus parenchyma can be easily identified by an experienced pediatric radiologist or endocrinologist. Diagnosis and follow-up of ectopic intrathyroidal thymic tissue can be performed noninvasively by thyroid ultrasound.

Ectopic juvenile intrathyroidal thymus presents as a solid and hypoechogenic area, with regular and sharp margins and no blood flow on power/color Doppler examination [1]. The thymus undergoes a process called involution, which is defined as a decrease in the size and weight of the gland with advancing age. During involution, the epithelial component undergoes atrophy, resulting in small scattered lymphocytes in abundant adipose tissue [3]. Ectopic thymus during involution appears as an hypoechogenic area with multiple hyperechogenic dots (microcalcification-like), and similar tissue is observed usually below the thyroid lower poles where the thymus is located [1]. Hyperechogenic dots observed in intrathyroidal ectopic thymus are pseudomicrocalcifications and can be

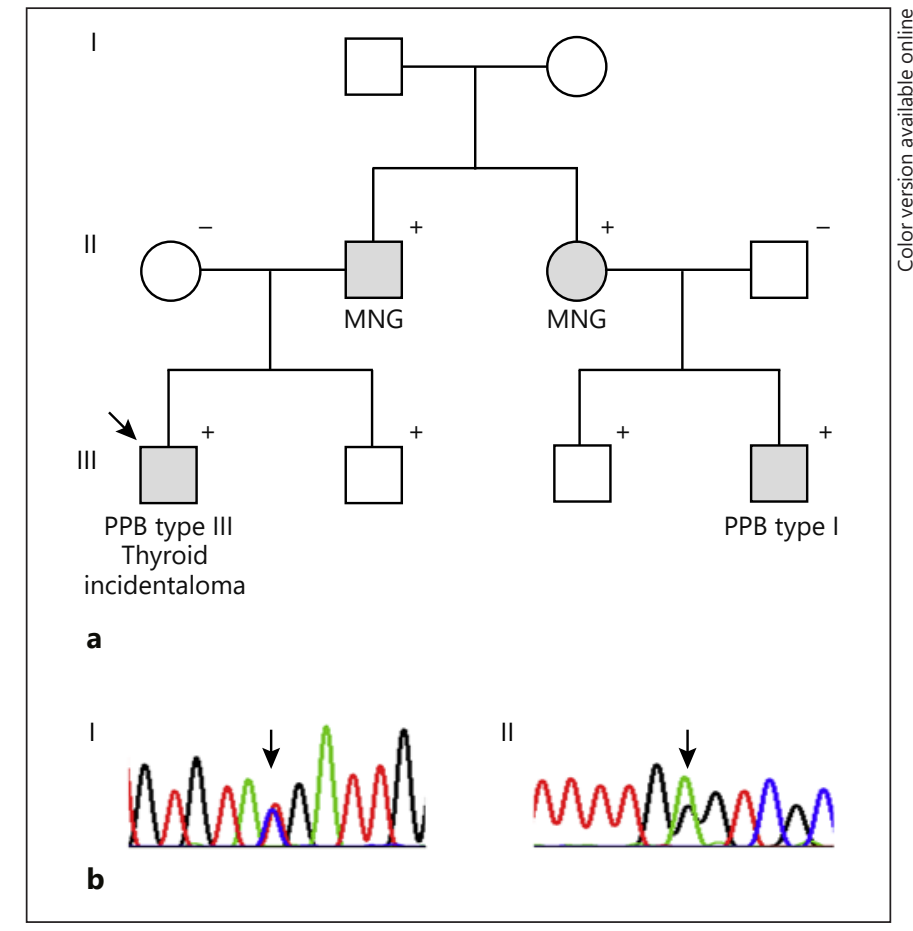

Fig. 1. a Pedigree of the family shows a remarkable family history. b Chromatograms show the germline DICER1 variant, c.2062C > T, p.R688* (panel I) and the somatic DICER1 mutation, c.5438A >G, p.E1813G, identified in the pleuropulmonary blastoma (PPB) (panel II). MNG, multinodular goiter.

mistaken for the true microcalcifications ( $<2 \mathrm{~mm}$ in diameter/size) observed in papillary thyroid microcarcinoma (mPTC) or medullary thyroid microcarcinoma (mMTC), thereby leading to a stressful clinical situation for the patient and physician. Intrathyroidal ectopic thymic tissue can thus be mistaken for a thyroid nodule and may lead to unnecessary surgery and/or invasive diagnostic procedures in children due to the overlap of its appearance with mPTC or mMTC on ultrasound. The widespread use of thyroid ultrasound in children can result in an increased detection rate of thymic inclusions, and, as such, ectopic intrathyroidal thymus should be considered in the differential diagnosis of an apparent thyroid nodule in children and adolescents [2].

Pleuropulmonary blastoma (PPB) is a hallmark component of DICER1 syndrome and diagnosis should prompt surveillance for other DICER1 syndrome-related disorders including multinodular goiter (MNG), cystic nephroma, pituitary blastoma, Sertoli-Leydig cell tumor, cervical embryonal rhabdomyosarcoma, and others [4-6]. The phenotypic spectrum of DICER1 syndrome is broad, but, apart from MNG, it generally includes rare-to-very-rare entities 

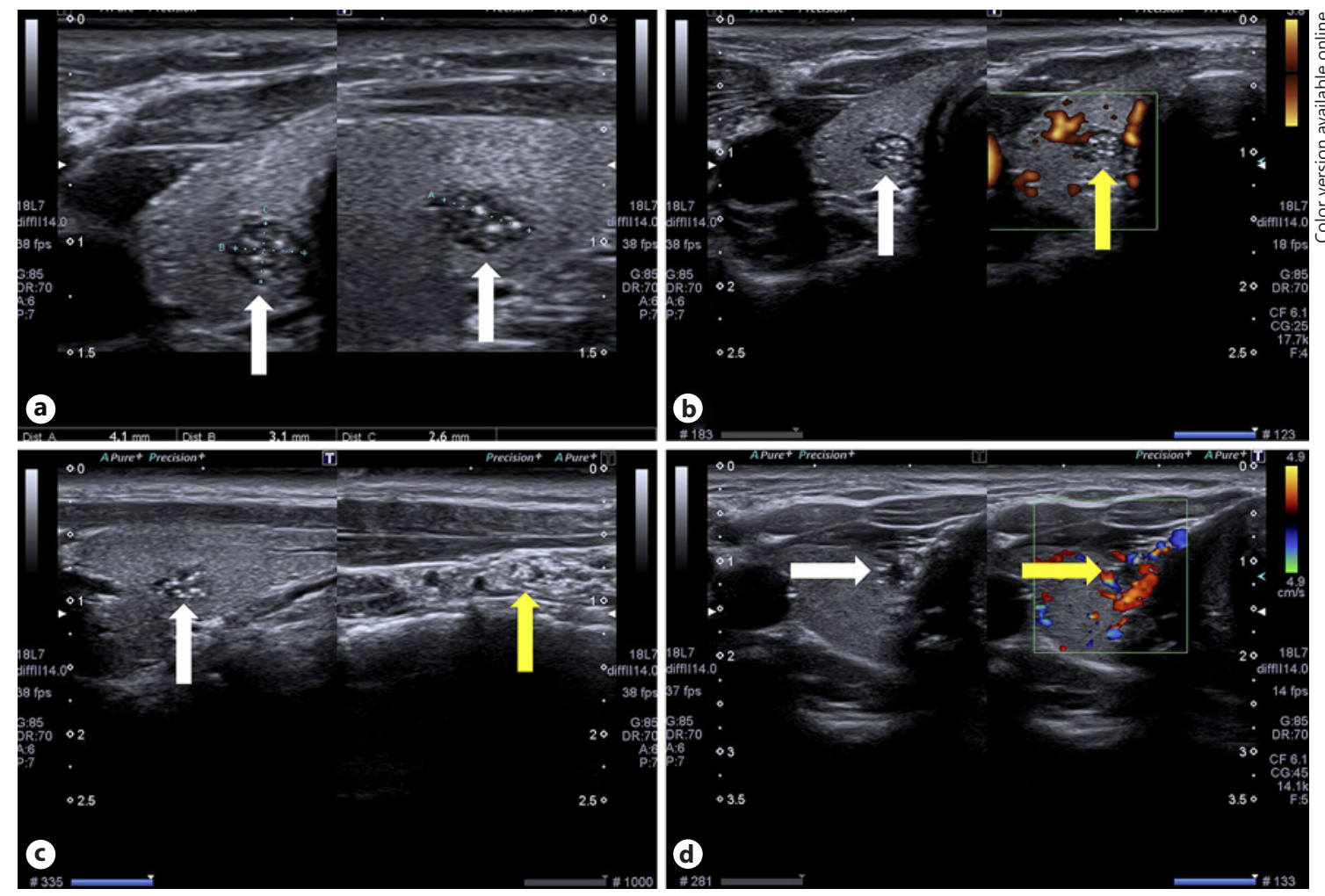

Fig. 2. Thyroid ultrasound images. a Intrathyroidal ectopic thymus in the right lobe (white arrows) in transverse and longitudinal projection. b A lack of blood flow within the lesion on power Doppler (yellow arrow; transverse projection). c Involuting thymus beneath the left lobe (yellow arrow; longitudinal projection) and intrathyroidal thymic lesion within the right lobe (white arrow; longitudinal projection). d Subcapsular papillary thyroid microcarcinoma from an unrelated patient presented as solid hypoechogenic lesion, with irregular borders, with a few microcalcifications (white arrow; transverse projection) and no blood flow on color Doppler (yellow arrow, transverse projection). This cancerous ultrasonographic suspicion of the "nonpalpable" thyroid nodule (incidentaloma) of the right lobe was verified postoperatively on histopathological sections.
[7]. DICER1 syndrome is caused by loss-of-function germline DICER1 alterations on chromosome 14q32.13 and exhibits an autosomal dominant pattern of inheritance [8]. Thyroid disease is the most frequent feature of DICER1 syndrome, particularly MNG, and affects approximately 75 and $20 \%$ of female and male heterozygotes, respectively, by the age of 40 years [9]. Rarely, papillary thyroid carcinoma [10] or follicular thyroid carcinoma [11] may occur in nonradiated DICER1 pathogenic variant heterozygotes, and it is also reported in those exposed to therapeutic radiation. Careful thyroid examination including ultrasound is thus necessary for DICER1 syndrome patients.

\section{Case Presentation}

A 4-year-old prepubertal boy was referred to the pediatric endocrinology department following an earlier diagnosis of PPB type III (solid type). The primary lung tumor was resected when the boy was aged 3 years and 4 months, and this was followed by chemotherapy. In view of the PPB diagnosis, genetic analysis was performed, which identified a germline DICER1 pathogenic variant (c.2062C>T, p.R688*) in the child. The father and paternal aunt (each of whom had MNG), the younger brother (who was unaffected), and 2 cousins (one of whom was unaffected and the other had PPB type I) were also heterozygous for the germline DICERI variant (Fig. 1). A second somatic DICER1 RNase IIIb hotspot mutation (c.5438A $>$ G, p.E1813G) was identified in DNA extracted from the proband's PPB (Fig. 1).

The patient's clinical thyroid examination was unremarkable. $\mathrm{He}$ was euthyroid with no goiter. $\mathrm{TSH}, \mathrm{fT}_{4}, \mathrm{fT}_{3}$, calcium, and calcitonin levels were all within the normal range. Thyroid ultrasound (Toshiba Premium Aplio 400) showed normal gland echogenicity, except for a $4.1 \times 3.1 \times 2.6 \mathrm{~mm}$ heterogenous lesion in the right lobe, which was solid and hypoechogenic with pseudomicrocalcifications (Fig. 2a). The lesion was well delineated and avascular on power Doppler (Fig. 2b). A similar but larger neck tissue area corresponding to involuting thymus was visible beneath the left lobe (Fig. 2c), supporting the diagnosis of an intrathyroid thymic lesion within the right lobe. A 3-year follow-up involving biannual clinical endocrine examination and thyroid ultrasound 
showed no change in the lesion size despite the patient's advancement in age, supporting the diagnosis of an ectopic thymus undergoing involution (Fig. 2a) but mimicking in part a true thyroid cancer in another patient (Fig. 2d).

\section{Discussion}

The increasing use of thyroid ultrasound for children may lead to a rise in the detection of thymic inclusions, and ectopic intrathyroidal thymus should be considered in the differential diagnosis of an apparent thyroid nodule in children and adolescents $[1,2,12]$. The thymus has a unique appearance on ultrasonography and ectopic thymus parenchyma can be easily identified. Diagnosis and follow-up of ectopic intrathyroidal thymic tissue can be performed noninvasively by thyroid ultrasound. The tissue may be considered substantially benign and self-limited if no change in size is observed over a period of months/ years; rarely, a small increase in size may be related to the normal growth of the thymus during development. In older patients, thymic inclusions exhibit a decrease in size and an increase in echogenicity, and the margins of the lesions become less defined, most likely reflecting normal thymus involution that occurs with advancing age and involves the atrophy of the epithelial component, resulting in small scattered lymphocytes in abundant adipose tissue [1, 2, 12]. To the experienced examiner, the appearance of thymic tissue is pathognomonic on ultrasound and is associated with stability or regression at follow-up. The prevalence of ectopic thymus detected incidentally in children who underwent neck or thyroid ultrasonography was $2.3 \%$ $(16 / 690)$ [13], lower than the previously reported 3.9\% (7/180) [14] and 17.3\% (9/52) [15]. This wide range in prevalence may have largely been due to the number of patients examined in each study. A prevalence of $8 \%$ of ectopic thymus occurring in young children with congenital hypothyroidism related to thyroid dysgenesis was reported [16]. For these patients, abnormal thymus development coexisted with a thymus in its normal location. The regression of intrathyroidal thymic inclusions can be safely monitored by ultrasound, thus avoiding invasive fine-needle aspiration biopsy (FNAB) or surgery. Tumors arising from the ectopic thymus are extremely rare and can also be monitored by ultrasound [12].

Despite the diagnosis of DICER1 syndrome in our patient, invasive diagnostic workup (FNAB under anesthesia) and surgical intervention of the thyroid incidentaloma were not indicated. We previously reported the case of a young patient whose parent had MEN2A [1]. The child was found to have an intrathyroidal ectopic thymus. The patient was negative for the RET mutation found in a parent, but concern led to full RET sequencing, which was negative. Thus, presence of the new thyroid lesion in this patient generated doubt as to whether the genetic testing result was correct and resulted in unnecessary additional genetic testing.

In the same vein, based on long-term experience in pediatric thyroid ultrasound, the diagnosis of ectopic thymic residue in the child with DICER1 syndrome was made in the absence of cytological and molecular tests that would necessitate invasive FNAB under general anesthesia. The appearance of the lesion was strongly consistent with intrathyroidal thymus on ultrasound, but indolent $\mathrm{mPTC}$ was not ruled out explicitly. The lack of enlargement over time also does not fully exclude mPTC. Ito et al. [17] showed the data of 1,235 patients with subclinical low-risk mPTC over an average observation period of 75 months. The rate of incidence of enlargement at the 5- and 10-year observations in this study was 4.9 and $8.0 \%$, respectively. Enlargement of mPTC in the youngest population $(<40$ years of age) at 5 years was noted in only $9.1 \%$ of the participants. In the remaining $90.9 \%$, the size of the mPTC remained unchanged. Unfortunately, such data on children $<10$ years of age are not available in the literature. The prevalence of ectopic intrathyroidal thymus in children is relatively high, in contrast to the extremely rare occurrence of microcancer in this age group. Despite this, it should be borne in mind that thyroid ultrasonography is not a test to confirm or exclude thyroid cancer. In summary, the case presented here exemplifies that thyroid incidentalomas in DICER1 syndrome patients should be viewed with great suspicion, but that not all thyroid disorders are DICER1related in these patients.

\section{Acknowledgments}

This work was supported by a Canadian Institutes of Health Research Foundation grant (FDN148390) to Dr. Foulkes. Dr. Niedziela was a visiting professor at McGill University, Montreal, QC, Canada in July and August 2018. His sabbatical leave was supported by the Poznan University of Medical Sciences and the Karol Jonscher's Clinical Hospital of Poznan University of Medical Sciences.

\section{Statement of Ethics}

This study was approved by the Institutional Review Board of the Faculty of Medicine of McGill University, Montreal, QC, Canada (Nos. A12-M117-11A and A08-M61-09B) and the Bioethical Commission on Human Research, Poznan University of Medical Sci- 
ences, Poznan, Poland (Nos. 257/01 and 258/01). Participants were recruited to the study in compliance with the second edition of the Canadian Tri-Council Policy Statement of Ethical Conduct of Research Involving Humans. The parents of subjects gave their written informed consent to publish the cases (including any images).

\section{Conflict of Interest Statement}

The authors have nothing to disclose.

\section{Author Contributions}

M.N. performed the clinical examination, referred the patient for genetic testing, conceived the study, and wrote the manuscript. N.S. performed the DICER1 genetic testing. L.d.K. assisted with genetic testing and the writing of the manuscript. W.D.F. oversaw the DICER1 genetic testing and reviewed the manuscript.

\section{References}

1 Niedziela M. Thyroid nodules. Best Pract Res Clin Endocrinol Metab. 2014 Mar;28(2):24577.

2 Segni M, di Nardo R, Pucarelli I, Biffoni M. Ectopic intrathyroidal thymus in children: a long-term follow-up study. Horm Res Paediatr. 2011;75(4):258-63.

3 Nishino M, Ashiku SK, Kocher ON, Thurer RL, Boiselle PM, Hatabu H. The thymus: a comprehensive review. Radiographics. 2006 Mar-Apr;26(2):335-48.

4 Rath SR, Bartley A, Charles A, Powers N, Baynam $G$, Jones T, et al. Multinodular Goiter in children: an important pointer to a germline DICER1 mutation. J Clin Endocrinol Metab. 2014 Jun;99(6):1947-8.

5 Rio Frio T, Bahubeshi A, Kanellopoulou C, Hamel N, Niedziela M, Sabbaghian N, et al. DICER1 mutations in familial multinodular goiter with and without ovarian Sertoli-Leydig cell tumors. JAMA. 2011 Jan;305(1):6877.

6 Slade I, Bacchelli C, Davies H, Murray A, Abbaszadeh F, Hanks S, et al. DICER1 syndrome: clarifying the diagnosis, clinical features and management implications of a pleiotropic tumour predisposition syndrome. J Med Genet. 2011 Apr;48(4):273-8.
7 Foulkes WD, Priest JR, Duchaine TF. DICER1: mutations, microRNAs and mechanisms. Nat Rev Cancer. 2014 Oct;14(10):66272.

8 Hill DA, Ivanovich J, Priest JR, Gurnett CA, Dehner LP, Desruisseau D, et al. DICER1 mutations in familial pleuropulmonary blastoma. Science. 2009 Aug;325(5943):965.

9 Khan NE, Bauer AJ, Schultz KA, Doros L, Decastro RM, Ling A, et al. Quantification of thyroid cancer and multinodular goiter risk in the DICER1 syndrome: a family-based cohort study. J Clin Endocrinol Metab. 2017 May; 102(5):1614-22.

10 Rutter MM, Jha P, Schultz KA, Sheil A, Harris $\mathrm{AK}$, Bauer AJ, et al. DICER1 mutations and differentiated thyroid carcinoma: evidence of a direct association. J Clin Endocrinol Metab. 2016 Jan;101(1):1-5.

11 Yoshida M, Hamanoue S, Seki M, Tanaka M, Yoshida K, Goto H, et al. Metachronous anaplastic sarcoma of the kidney and thyroid follicular carcinoma as manifestations of DICER1 abnormalities. Hum Pathol. 2017 Mar; 61:205-9.
12 Kay-Rivest E, Mascarella MA, Puligandla P, Emil S, Saint-Martin C, Nguyen LH, et al. Intrathyroidal thymic tissue in children: avoiding unnecessary surgery. J Pediatr Surg. 2018 May;53(5):1010-3.

13 Bang MH, Shin J, Lee KS, Kang MJ. Intrathyroidal ectopic thymus in children: A benign lesion. Medicine (Baltimore). 2018 Apr; 97(14):e0282.

14 Kabaalioğlu A, Öztek MA, Kesimal U, Çeken K, Durmaz E, Apaydın A. Intrathyroidal ectopic thymus in children: a sonographic survey. Med Ultrason. 2017 Apr;19(2):179-84.

15 Avula S, Daneman A, Navarro OM, Moineddin R, Urbach S, Daneman D. Incidental thyroid abnormalities identified on neck US for non-thyroid disorders. Pediatr Radiol. 2010 Nov;40(11):1774-80.

16 Bubuteishvili L, Garel C, Czernichow P, Léger J. Thyroid abnormalities by ultrasonography in neonates with congenital hypothyroidism. J Pediatr. 2003 Dec;143(6):759-64.

17 Ito Y, Miyauchi A, Kihara M, Higashiyama T, Kobayashi K, Miya A. Patient age is significantly related to the progression of papillary microcarcinoma of the thyroid under observation. Thyroid. 2014 Jan;24(1):27-34. 